seizures themselves is uncommon, a finding that should help to allay the fear often expressed by parents of a child with a first convulsion.

Camfields' findings are similar to those of Callenbach PMC et al who reported a mortality rate of 3.8/1000 person-years, seven times higher than expected in a cohort of 472 children in the Netherlands. None of 328 children with nonsymptomatic epilepsy died. In those with symptomatic epilepsy, the mortality risk was 22.9 vs 0.39 expected in a control population (Pediatrics 2001;107:12591263; see Ped Neur Briefs June 2001;15:43-44). Among the 9 deaths in this prospective study, none was sudden unexpected and unexplained (SUDEP). SUDEP risk factors are usually multiple and include early-onset epilepsy, poor seizure control, polytherapy with antiepileptic drugs (AED), and frequent dose adjustments or abrupt AED withdrawal (Nilsson et al. In Gordon N. Dev Med Child Neurol 2001;43:354-357).

An accurate initial diagnosis is important in counseling parents regarding risk of mortality in childhood epilepsy. Children with secondarily generalized epilepsy associated with functional neurological deficit are particularly at risk of a poor prognosis. Apart from the rare exception of SUDEP in children with tuberous sclerosis, deaths in epilepsy are usually explained.

\title{
FOCAL CORTICAL HYPOMETABOLISM AND INFANTILE SPASMS
}

The occurrence and prognostic significance of focal defects in cerebral cortical glucose metabolism were evaluated in infants with newly diagnosed symptomatic and cryptogenic infantile spasms examined at Turku and Helsinki Universities, Finland. MRI, video-EEG, and PET were obtained within 2 weeks of diagnosis in 10 patients with symptomatic and 7 with cryptogenic infantile spasms. Twelve patients had repeat PET at 1 year of age. Cortical hypometabolic foci occurred in 13 (77\%) infants; 7 had symptomatic and 6 cryptogenic spasms. In 7 of 9 reexamined with PET at age 1, foci had disappeared, and none of 6 with occipital foci had persistent hypometabolism. The focal PET abnormalities correlated with focal findings on video-EEG. Quantitative cortical and subcortical glucose metabolic rates were similar in cryptogenic and symptomatic spasms at onset. Striatal values are higher than cortical values in all patients. Focal lesions in glucose metabolism associated with infantile spasms have no prognostic value for seizure outcome. (Metsahonkala L, Gaily E, Rantala H et al. Focal and global cortical hypometabolism in patients with newly diagnosed infantile spasms. Neurology, June (1 of 2) 2002;58:1646-1651). (Reprints: Dr L Metsahonkala, Department of Pediatrics, Turku University Central Hospital, PL52 20521 Turku, Finland).

COMMENT. Cortical hypometabolic lesions demonstrated by PET in children with infantile spasms are usually transient, especially those in occipital regions, the most common localization. They are equally common in cryptogenic and symptomatic spasms. PET and video-EEG abnormalities are correlated, but PET is the better test to detect focal cortical dysfunction. PET is of no value in prognosis, whereas infants with MRI structural abnormalities are at increased risk of continuing seizures and poor developmental outcome. Most children with infantile spasms and favorable outcome have transient foci of cortical hypometabolism at onset, especially occipital in location.

Bitemporal glucose hypometabolism may be indicative of a poor prognosis, delayed development, severe dysphasia, and autism in children with infantile spasms examined by PET. (Chugani HT et al. 1996; see Progress in Pediatric Neurology III, PNB Publ, 1997;pp40-41). 